Abstract 22 Table 1 Clinical characteristics and clinical outcomes

\begin{tabular}{llll}
\hline & $\begin{array}{l}\text { Nuclear BAP1 IHC } \\
\text { positive } \\
(\mathbf{n}=11)\end{array}$ & $\begin{array}{l}\text { Nuclear BAP1 IHC } \\
\text { negative } \\
(\mathbf{n}=66)\end{array}$ & $\begin{array}{l}\mathbf{p} \text { - } \\
\text { value }\end{array}$ \\
\hline $\begin{array}{l}\text { Gender (M=male) } \\
\text { Median age at diagnosis (years) }\end{array}$ & 69.5 & M: $91 \%$ & 0.30 \\
Histology & & 66.0 & 0.94 \\
Epithelioid & $82 \%$ & & 0.57 \\
Biphasic & $18 \%$ & $89 \%$ & \\
Sarcomatoid & $0 \%$ & $9 \%$ & \\
Median overall survival from & & $3 \%$ & 0.22 \\
diagnosis (months) & & & 0.62 \\
All & 21.0 & 23.3 & 0.11 \\
Active symptom control (ASC) & 24.3 & 25.0 & 0.69 \\
ASC+vinorelbine & 12.8 & 22.8 & \\
ASC+mitomycin, vinblastine, & 15.7 & 19.4 & \\
cisplatin & & & \\
\hline
\end{tabular}

\section{S23 EVALUATION OF PHOSPHORYLATED PROTEIN KINASE B (AKT) AND MAMMALIAN TARGET OF RAPAMYCIN (MTOR) EXPRESSION IN MALIGNANT PLEURAL MESOTHELIOMA (MPM) AND THEIR ASSOCIATION WITH PATIENT SURVIVAL-A RETROSPECTIVE COHORT STUDY}

${ }^{1} S$ Tariq, ${ }^{2} \mathrm{~A}$ Campbell, ${ }^{3} \mathrm{~L}$ Cawkwell, ${ }^{4} \mathrm{MJ}$ Lind. ${ }^{1}$ Hull York Medical School, Hull, UK; ${ }^{2}$ Cellular Pathology Department, Hull and East Yorkshire Hospitals NHS Trust, Hull, UK; ${ }^{3}$ School of Life Sciences, University of Hull, Hull, UK; ${ }^{4}$ Hull York Medical School and Hull and East Yorkshire Hospitals NHS Trust, Hull, UK

\subsection{6/thoraxjnl-2017-210983.29}

Background Dysregulation of the PI3K/AKT/mTOR pathway has been observed in various cancers and has also been suggested to be involved in mesothelioma. In some cancers, a significant association has been found between the expression of these proteins and patient survival. In this study, we aimed to investigate the expression of phosphorylated AKT/mTOR in our archival MPM tissue samples and evaluate their relationship with patient survival.

Methods Immunohistochemistry was performed on 82 archival MPM tissue samples to examine the expression of phosphorylated AKT/mTOR. Histopathological and clinical data of relevant patients were obtained from Hull Royal Infirmary. Mesothelioma tissues with $>25 \%$ staining were grouped as positive while tissues with $<25 \%$ staining were grouped as

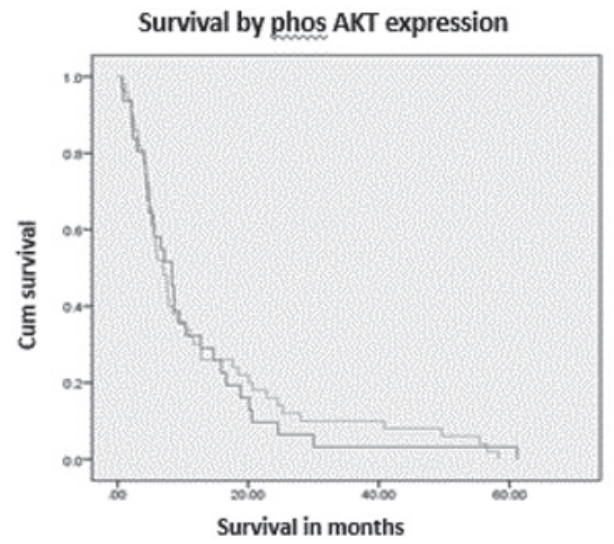

negative. Colorectal cancer tissues were used as positive and negative controls. Univariate analysis for protein expression and histological subtypes was performed using Kaplan Meier survival curves with log rank analysis. Multivariate Cox regression analysis taking histological subtypes into account was performed, to assess the effect of phosphorylated AKT/mTOR expression on patient survival.

Results Our data set included 44 epithelioid, 24 biphasic and 14 sarcomatoid tissue samples. Of the MPM tissues samples, $63.41 \%$ demonstrated positive expression for phosphorylated mTOR protein while $61.73 \%$ showed positive phosphorylated AKT expression. We did not observe a significant difference in expression of phosphorylated AKT/mTOR between the histological subtypes of MPM $(p>0.05)$. Positive expression of phosphorylated AKT/mTOR proteins was not associated with survival in Kaplan Meier survival curve analysis $(\mathrm{p}>0.05)$. When histological subtypes were taken into account, multivariate Cox regression analysis demonstrated that neither phosphorylated mTOR nor phosphorylated AKT expression were independent prognostic factors for survival $(\mathrm{p}>0.05)$.

Conclusion Our data suggest that phosphorylated AKT/mTOR are expressed in a significant proportion of MPM samples. However, no statistically significant association was found between phosphorylated AKT/mTOR/expression and patient prognosis.

\section{REFERENCE}

1. Li S, Wang Z, Huang J, Cheng S, Du H, Che G, Peng Y. Clinicopathological and prognostic significance of mTOR and phosphorylated mTOR expression in patients with esophageal squamous cell carcinoma: A systematic review and meta-analysis. BMC Cancer 2016;16(1):877.

\section{S24 A PHASE I FEASIBILITY STUDY IN ESTABLISHING THE ROLE OF ULTRASOUND-GUIDED PLEURAL BIOPSIES IN PLEURAL INFECTION (THE AUDIO STUDY)}

${ }^{1}$ I Psallidas, ${ }^{1} \mathrm{~N}$ Kanellakis, ${ }^{2} \mathrm{R}$ Bhatnagar, ${ }^{1} \mathrm{R}$ Ravindran, ${ }^{1} \mathrm{~A}$ Yousuf, ${ }^{3} \mathrm{AJ}$ Edey, ${ }^{1} \mathrm{RM}$ Mercer, ${ }^{1} \mathrm{JP}$ Corcoran, ${ }^{1} \mathrm{RJ}$ Hallifax, ${ }^{1} \mathrm{P}$ Shetty, ${ }^{4} \mathrm{~T}$ Dong, ${ }^{5} \mathrm{HEG}$ Piotrowska, ${ }^{6} \mathrm{C}$ Clelland, ${ }^{2} \mathrm{NA}$ Maskell, ${ }^{1} \mathrm{NM}$ Rahman. ${ }^{1}$ Oxford Centre for Respiratory Medicine, Churchill Hospital, Oxford University Hospitals NHS Foundation Trust, Oxford, UK; ${ }^{2}$ Academic Respiratory Unit, School of Clinical Sciences, University of Bristol, Bristol, UK; ${ }^{3}$ Department of Radiology, Southmead Hospital, North Bristol NHS Trust, Bristol, UK; ${ }^{4}$ Medical Research Council Human Immunology Unit, Medical Research Council Weatherall Institute of Molecular Medicine, Raddliffe Department of Medicine, Oxford, UK; ${ }^{5}$ Oxford Respiratory Trials Unit, Nuffield Department of Medicine, University of Oxford, Oxford, UK; ${ }^{6}$ Department of Cellular Pathology, Oxford University Hospitals NHS Foundation Trust, Oxford, UK

\subsection{6/thoraxjnl-2017-210983.30}

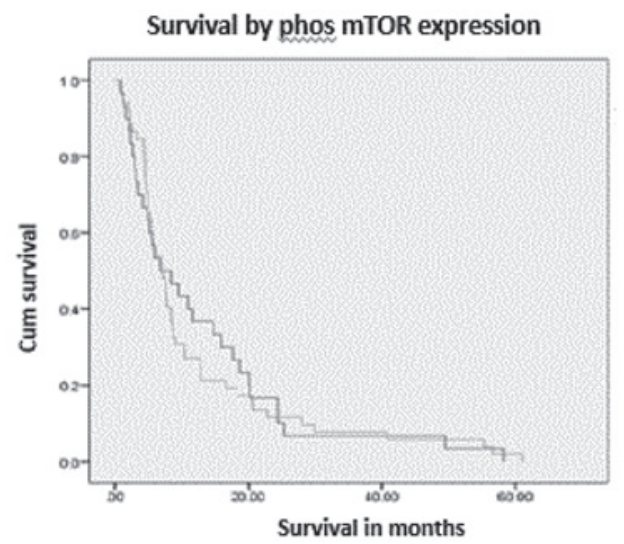

Abstract 23 Figure 1 (A) Survival by phos AKT expression and (B) Survival by phos mTOR expression. 

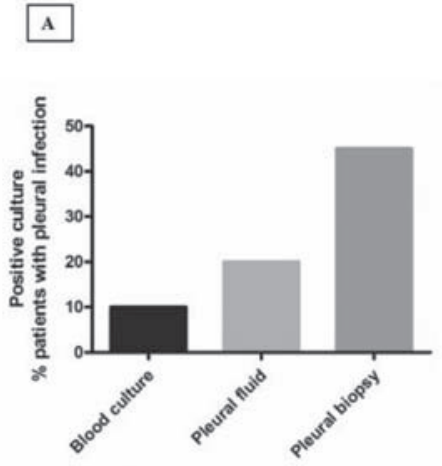

B

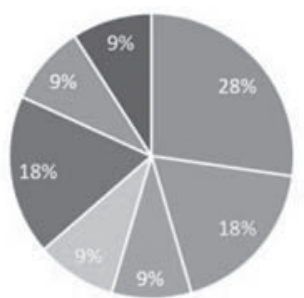

- Streptococcus milleri = Streptococcus intermedius
= Klebsiella pneumoniae = Anaerobes
- Staphylococcus aureus = Staphylococcus lugdunesis
- Staphylococcus Epidermidis

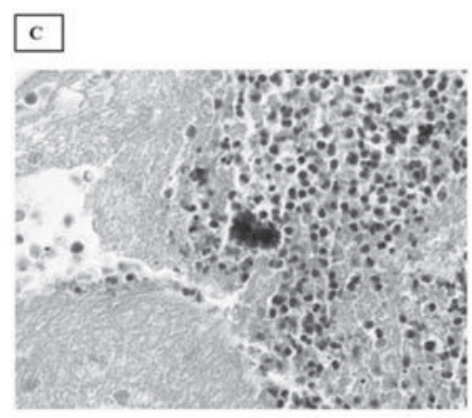

Abstract 24 Figure 1 (A)\% increase on positive culture samples in patients with pleural infection, (B) Results of pleural biopsy culture, and (C) Gram stain of acute inflammatory exudate in a pleural biopsy showing small colonies of Gram positive cocci.

Background Pleural infection is a common complication of pneumonia associated with high mortality and poor clinical outcome. Treatment of pleural infection relies on the use of broad-spectrum antibiotics, since reliable pathogen identification occurs infrequently. We performed a feasibility interventional clinical trial assessing the safety and significance of ultrasound (US)-guided pleural biopsy culture to increase the microbiological yield.

Methods 20 patients with clinically established pleural infection were recruited. Participants underwent a detailed US scan and US-guided pleural biopsies before chest drain insertion, alongside standard clinical management. Pleural biopsies and routine clinical samples (pleural fluid and blood) were submitted for microbiological analysis. In an exploratory sub-study, the 16S rRNA technique was applied on pleural biopsy samples, to investigate its' utility on increasing speed and accuracy versus standard microbiological diagnosis. This trials is registered with ClinicalTrials.gov, number NCT02608814

Findings US-guided were safe with no adverse events observed in this study. Pleural biopsies increased microbiological yield by $30 \%$ in addition to pleural fluid and blood samples (combined diagnostic sensitivity 55\%). US characteristics at baseline were not statistically associated with survival, fluid volume drainage, radiological improvement or need for surgery. The $16 \mathrm{~S}$ rRNA technique was successfully applied to pleural biopsy samples, demonstrating high sensitivity (93\%) and specificity (89.5\%).

Conclusion Our findings demonstrate safety of conducting US guided biopsies in patients with pleural infection and a substantial increase in microbiological diagnosis. qPCR primer assessment of pleural fluid and biopsy appears to have excellent sensitivity and specificity.

Funding Oxfordshire Health Services Research Committee

\section{S25 EOSINOPHILIC PLEURAL EFFUSIONS - A LARGE PROSPECTIVE STUDY ON AETIOLOGY AND PROGNOSIS}

${ }^{1} \mathrm{~S}$ Walker, ${ }^{1} \mathrm{~A}$ Morley, 'L Stadon, ${ }^{1} \mathrm{~N}$ Zahan-Evans, ${ }^{2} \mathrm{~A}$ Medford, ${ }^{1} \mathrm{~N}$ Maskell. ${ }^{1}$ Academic Respiratory Unit, Bristol, UK; ${ }^{2}$ Southmead Hospital, Bristol, UK

\subsection{6/thoraxjnl-2017-210983.31}

Introduction and Objectives Eosinophilic pleural effusions (EPE) are a relatively uncommon finding in the investigation of undifferentiated pleural effusions. Traditionally defined as a cell count $\geq 10 \%$ eosinophils, it was initially felt to be a
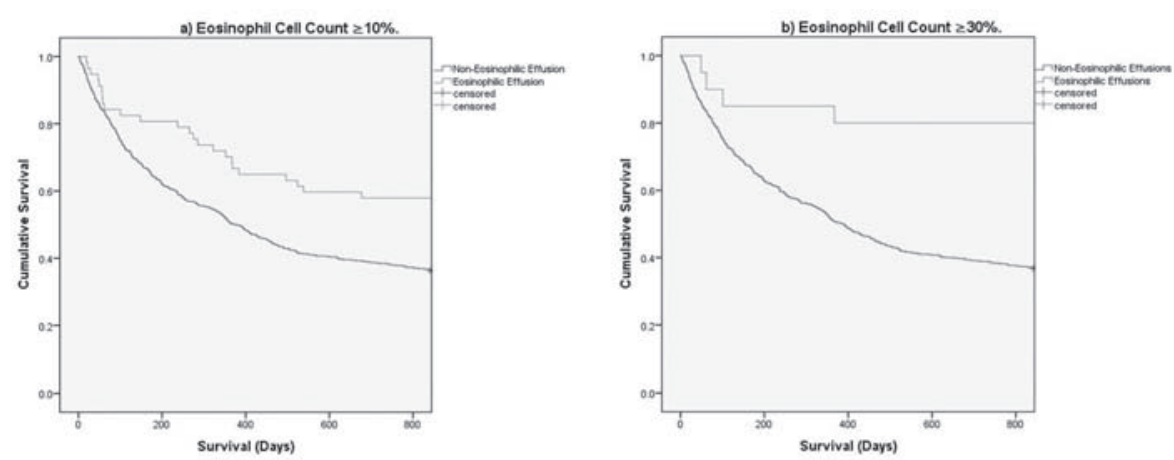

\begin{tabular}{|c|c|c|c|c|c|c|c|c|c|}
\hline \multicolumn{10}{|c|}{ Numbers at risk } \\
\hline 57 & 46 & 37 & 34 & 33 & 20 & 17 & 16 & 16 & 16 \\
\hline \multicolumn{10}{|c|}{ Non-Eosinophilic Effusion } \\
\hline 746 & 463 & 360 & 302 & 272 & 783 & 492 & 381 & 320 & 282 \\
\hline
\end{tabular}

Abstract S25 Figure 1 Kaplan-meier survival curves for Eosinophilic vs Non-Eosinophilic effusions. 